\title{
Developing a software Architecture Comparison Analysis Method for Critical Systems at International Airports
}

\author{
Shady Gomaa Abdulaziz \\ safaga_diadora@hotmail.com \\ College of Computing \& Information \\ Technology, Arab Academy
}

\author{
Ahmed El-Abbassy \\ ahmed_elabbassy@yahoo.com \\ Higher Institute of Computer Science\& \\ Information Technology, \\ El-Shorouk Academy
}

\author{
Abdelfatah A. Hegazy \\ abdheg@hotmail.com \\ College of Computing \& \\ Information Technology, Arab \\ Academy
}

\begin{abstract}
Software Architecture Comparison Analysis Methods provide organizations with a rationale for an architecture selection process by comparing the fitness of software architecture candidates for required systems. Comparing software architectures for any nontrivial system is a difficult task. Software architectures are designed with particular requirements and constraints, and are often poorly documented.

With the lack of data about software architecture, developing comparison methods based on a black box approach is considered very helpful, and architectures can be compared based on a set of criteria derived from the business goals of an organization.
\end{abstract}

A popular method for comparing software architectures as black box is the Software Architecture Comparison Analysis Method (SACAM) developed by the Software Engineering Institute (SEI). SACAM compares the architectures of software systems and not the implementation code. SACAM does not address enterprise architecture issues such as the implemented software evolution and maintenance.

This paper discusses and presents a proposed adaptation of SACAM to be applied in the context of critical socio-technical systems where issues of architecture evolution and maintenance are considered important factors in selecting a strategy for software modernization. The proposed method is called software Architecture Comparison Analysis Method for Critical Systems (SACAM-CS). SACAM-CS is an architecture selection method based on multi-criteria decision analysis. The proposed method has been validated using a suitable case study to compare among two check-in systems used in international airports. The purpose of the case study is to investigate, examine, analyze, and develop model architecture to compare between two check-in systems with regard to evaluation of process improvement. 
Moreover, the case study aims to analyzing the impacts of conventional check-in system and self-Service Technology at Cairo International Airport through identification of measures and methods for success evaluation. The main methodology is to use empirical data from Cairo International Airport accompanied by research, as an input for suggesting proposals for improvement and discussing new model architecture for future research in Aeronautical software not addressed by e.g. traditional comparative analysis of software theory or comparative study of software architecture analysis research.

\section{Key words:}

Software Engineering, Software Architecture Comparison, Software Evolution, Legacy Software, Socio-Technical Systems, Airport Check-in Systems.

\section{Introduction}

Critical systems are technical or socio-technical systems that people or businesses depend on. If these systems fail to deliver their services as expected then serious problems and significant losses may result. Typically there are three main categories of critical systems: Safety-critical systems, Mission-critical systems and Business-critical systems [1]. Socio-Technical systems are systems that include hardware, software components, procedures and operational processes.

The most important emergent property of a critical socio-technical system is its dependability. Dependability was proposed to cover the related system attributes of availability, reliability, safety and security [2]. Reliability and availability are usually considered to be the most important dimensions of dependability.

Critical Socio-Technical systems are complex systems usually with a long lifetime [3]. Its development continues throughout its life with changes to accommodate with new requirements, new operating platforms, and so forth. With time SocioTechnical software becomes legacy software that has been developed in the past using older or obsolete technology. Legacy software is often business-critical software, maintained because it is too risky to replace it. Modernizing legacy software should be based on periodical assessment in order to decide the most appropriate strategy for evolving these systems. Assessment should include both business value assessment and system quality assessment by measuring factors such as performance, interoperability, failure rate, and maintenance costs. As illustrated in figure 1 system stakeholders have typically four strategic options (choices) when considering software modernization. 


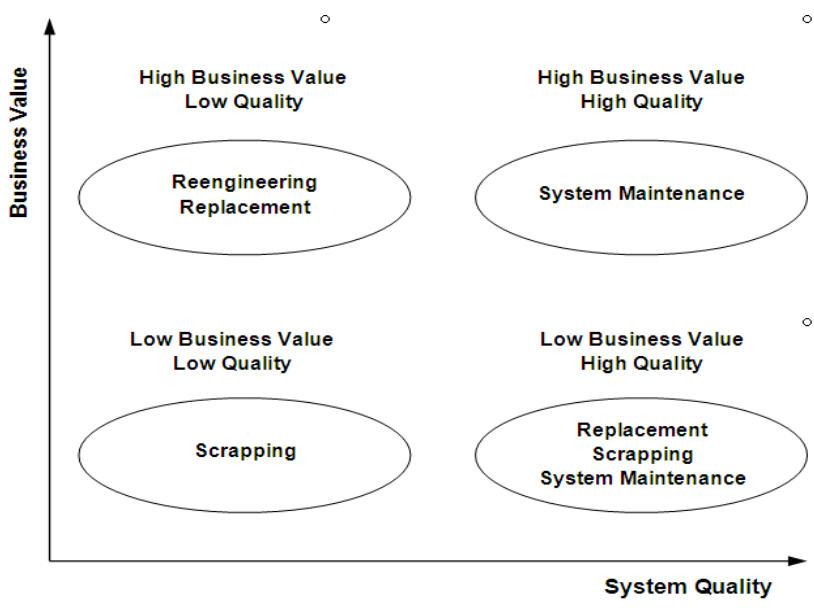

Figure 1: Legacy system clusters \& modernization options

Software Architecture Comparison Analysis Methods are used in the analysis and comparison among software products, and are used in software modernization situation assessment. These methods provide organizations with a rationale for an architecture selection process by comparing the fitness of software architecture candidates being used in envisioned systems [4].

The software architecture of a system is defined as the structure or structures of the system, which comprise software components, the externally visible properties of those components, and the relationships among them [26].

Software architectural evaluation can be conducted at different phases of software life cycle. Software architecture can be evaluated before its implementation (early evaluation), or after its implementation (late evaluation) [4, 20, 23]. Early software architectural evaluation can be conducted on the basis of the specification and description of the software architecture, and other sources of information, such as interviews with architects. Late software architectural evaluation is performed based on metrics $[20,22,25]$ and can be used for evaluation of existing systems before future maintenance or enhancement of the system as well as for identifying architectural drift and erosion[20, 22]. Software architectures are often poorly documented and with the lack of data about software architecture, developing evaluation methods based on black box approach is considered to be very helpful [24].

For both early and late software architectural evaluations, two basic categories are suggested [15]: qualitative evaluation and quantitative evaluation. Qualitative 
evaluation generates qualitative questions about software architecture to assess any given quality, whereas quantitative evaluation uses quantitative measurements taken from software architecture to address specific software qualities.

Techniques for generating qualitative questions include scenarios, questionnaires, and checklists. Scenarios appear to be the most utilized form of acquiring data [16]. There are also empirically-based approaches $[17,21]$ that define some relevant metrics for software architecture evaluation. The metrics are defined based on the goal of evaluation.

A popular Scenario-based, early evaluation method is the Software Architecture Comparison Analysis Method (SACAM) developed by the Software Engineering Institute (SEI) [5]. SACAM uses several architecture techniques developed by SEI to compare architecture candidates. SACAM compares the architectures of software systems and not the implementation code.

Software can be compared on several different levels. It is possible to compare the requirements, but that does not address how well the software actually realizes the requirements. On the other hand, at the implementation level, it is clear how well requirements are fulfilled, but comparing different software is almost impossible because of the huge amount of information. Comparing software architectures provides a manageable level of information.

SACAM does not address enterprise architecture issues such as implemented software evolution and maintenance. These issues are discussed in this paper where a proposed adaptation of SACAM is presented. The proposed method is called Software Architecture Comparison Analysis Method for Critical Systems (SACAM-CS). SACAM-CS will be applied in the context of critical sociotechnical systems where architecture evolution and maintenance are considered important factors in selecting a strategy for software modernization. SACAM-CS is an architecture selection method based on multi-criteria decision analysis. The proposed method has been validated using a suitable case study to compare between two check-in systems used in international airports.

The rest of this work is structured as follows: Section 2 presents an overview of SACAM. In section 3 SACAM-CS is discussed before describing the case study and the experiment in Sections 4, 5 and Section 6 describes the conclusion and future work on this topic.

\section{SACAM Overview}

Software Architecture Comparison Analysis Method (SACAM) provides organizations with a rationale for an architecture selection process by comparing the fitness of software architecture candidates being used in envisioned systems. The method addresses organizations that select software architectures to: 
1) Explore the support of existing software product architectures for required software product lines.

2) Decide among competitive software architectures proposed by subcontractors.

Comparing software architectures for any nontrivial system is a difficult task. Software architectures are designed with particular requirements and constraints, and are often poorly documented. However, organizations often need to select a software architecture for future development from several candidate architectures. Software Architecture Comparison Analysis Method (SACAM) was created to provide the rationale for an architecture selection process by comparing the fitness of architecture candidates for required systems. SACAM compares architectures based on a set of criteria derived from the business goals of an organization. SACAM was developed in a technical reuse context where an organization architectural commonalities and differences are investigated to explore architectural designs for software product line architecture.

SACAM is an early evaluation, Scenario-based method that was created to provide the rationale for an architecture selection process by comparing the fitness of architecture candidates for required systems [5].

The comparison is performed in a series of steps as follows:

Step 1 (Preparation) examines the available inputs to prepare a successful application of the method. The inputs to the method include: (1) architecture candidates - the architectures that should be compared; (2) business goals - the source of the comparison criteria.

Step 2 (Criteria Collation), a set of criteria for the architecture comparison is identified. A criterion formulates a requirement for the architecture to support the organization's business goals. Criteria are refined into quality attribute scenarios.

Step 3 (Determination of Extraction Directives) determines the architectural views, tactics, styles, and patterns that are looked for during the following extractions to find supporting evidence for the scenarios of Step2.

Step 4 (View and Indicator Extraction) extracts the architectural views for each candidate according to the extraction directives from step 3; detects indicators that support the quality attribute scenarios from Step 2. Architecture recovery techniques may be needed to generate relevant views.

Step 5 (Scoring), each criterion is scored for an architecture candidate. The scoring is based on the evidence provided by Step 4, and the quality attribute scenarios determined during step 2 . The scoring might consider weights that are provided by stakeholders for the criteria. The scoring provides the reasoning and the resulting score for how well the criteria scenarios are supported by a candidate. 
Step 6 (Summary) summarizes the results of the analysis.

SACAM uses techniques as illustrated in Figure 2. Each technique contributes to the comparison of software architectures.

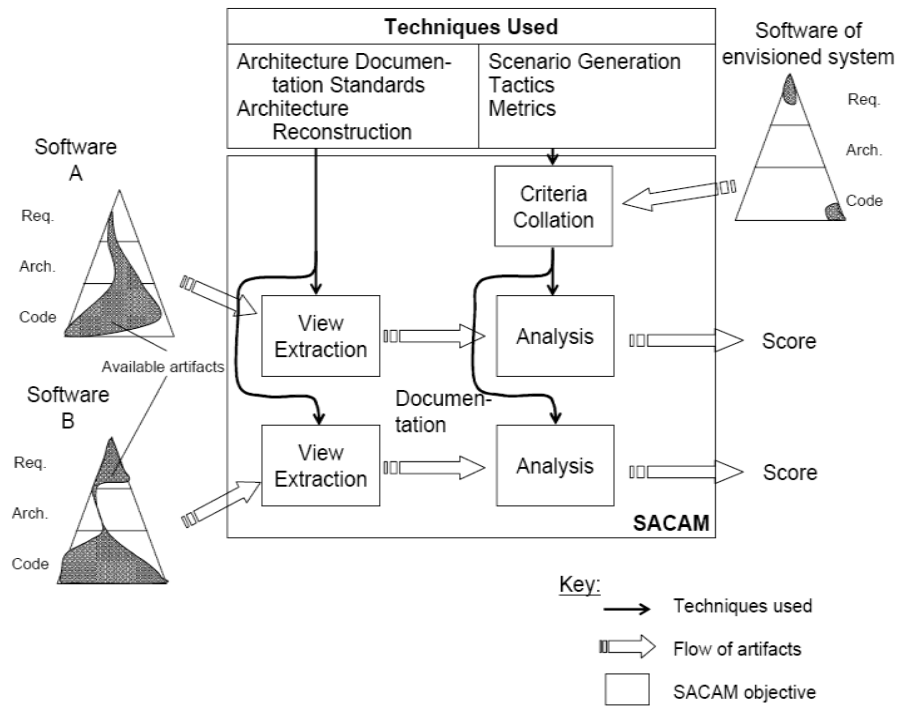

Figure 2: Architecture Techniques used by the SACAM

These techniques are used to generate the necessary artifacts that are then analyzed to provide the final scores for each architecture. SACAM techniques are summarized hereafter.

1) Scenario Generation: SACAM requires criteria that are derived from the business goals of an organization for an envisioned system. The criteria are articulated in quality attributes that are further refined into quality attribute scenarios. Scenario generation is a technique for capturing quality attributes and refining them into quality attribute scenarios. SEI methods that incorporate scenarios generation are the architecture tradeoff method [6] and the quality attribute workshop(QAW)[7].

2) Tactics: To achieve particular qualities that are addressed with scenarios, the notion of tactics strategies to achieve quality attribute goals is introduced[8]. SACAM uses tactics in the analysis as indicators to evaluate if the extracted views support a criterion articulated as a quality attribute scenario. Collections of tactics are available for a variety of quality attributes[9].

3) Metrics: Metrics support quantitative analysis that provides useful indicators of the overall complexity where change might be either difficult or most likely. 
Developing a software Architecture Comparison Analysis Method for Critical Systems at International Airports

Metrics are used in SACAM on the code level, if available, or on a detailed design level[10,11].

4) Architectural Documentation Standards: SACAM requires the availability of architectural documentation to perform the comparison criteria analysis. Experience shows that architectural documentation across systems is heterogeneous. For example, there are differences in notations, stakeholders, level of documentation detail, and scope. One of the SACAM's challenges is to obtain comparable architectural documentation. SACAM uses the "views and beyond" architectural documentation approach[12].

5) Architecture Reconstruction: The architectural documentation used to perform the comparison might be unavailable, insufficient, or out of date. In these cases the architecture has to be reconstructed. However not all architectural views have to be reconstructed, only the relevant views. For example if the intension is to find the architecture that is best suited to support modifiability, views showing module dependencies are more important than process views. This goal-oriented approach is used in the Quality-Attribute-driven Software Reconstruction (QADSAR) method [13].

The outputs of SACAM include a recommendation for the decision-making process, the scores and the related reasoning for each candidate, and the generated artifacts such as architectural views, tactics and scenarios.

SACAM is a standard framework that allow for comparing several architectures. This important feature permits, with some adaptation, to the use of SACAM in both early architecture evaluation (before its implementation) and late architecture evaluation (after its implementation).

The proposed adaptation is described in the next section.

\section{SACAM-CS}

Critical systems are systems in which defects could have a dramatic impact on human life, the environment or significant assets. Such systems are expected to satisfy a variety of specific qualities including reliability, availability, security and safety. With the steady infiltration of computers and software in all aspects of our modern world, critical systems increasingly depend on software functionality. These systems are commonplace in many different products, ranging from aircraft systems to home use medical devices.

The various stakeholders' quality requirements need to be considered collectively to describe the quality requirements of the envisioned system and therefore build the basis for the comparison and selection criteria. Given the impact that software architecture has on a project's success, the need to choose the right architecture assumes significance. In this chapter, a new architecture selection method based on 
multi-criteria decision analysis have been developed and validated using a suitable case study.

The Software Architecture Comparison Analysis Method for Critical System (SACAM-CS) provides airports and stakeholders' with a rationale for an architecture selection process by comparing the performance of software architecture between (CUTE and CUSS) candidates being used in envisioned systems or evolutes the CUTE or CUSS System that meets all needs of decision maker.

SACAM-CS is a proposed adaptation of SACAM that permits SACAM to be applied, not only as an early architecture evaluation method, but also as a late architecture evaluation method.

Late software architecture evaluation can use data measured on the implementation of software architecture, and metrics can be used to reconstruct the actual software architecture, allowing it to be compared to a planned architecture[17, 18, 19].

SACAM-CS complements the steps and techniques used by SACAM in order to allow for comparison among implemented software products, and consequently could be used in software modernization situation assessment.

AS illustrated in figure 3, the evolution/maintenance history is included in the comparison and analysis of candidate architectures. The rationale for including the evolution/ maintenance history is that implemented Software systems undergo constant change causing the architecture of the system to degenerate over time.

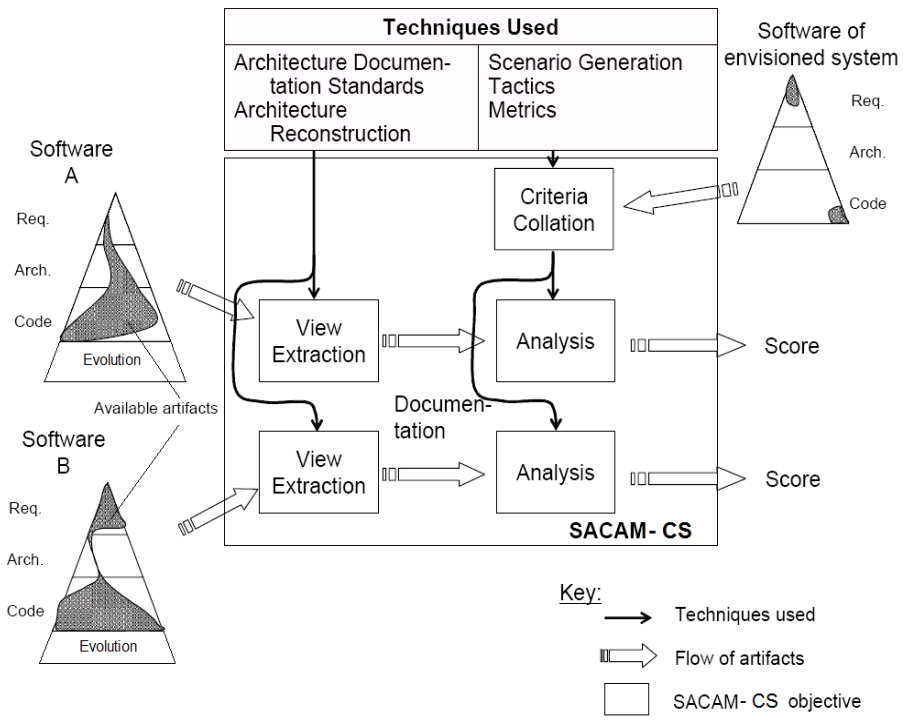

Figure 3: Architecture Techniques used by the SACAM 
Developing a software Architecture Comparison Analysis Method for Critical Systems at International Airports

In what follows the complementary techniques proposed by SACAM-CS are presented.

\subsection{Scenario Generation}

SACAM-CS will be applied to compare between two implemented systems. The goal of the scenario is to show which system is more reliable, efficient and achieves all quality attribute conditions by using quality attribute scenarios such as:

(1) Availability is concerned with system failure and duration of system failures.

(2) Modifiability is concerned with cost of change, both in time and money.

(3) Performance is concerned with response time.

(4) Security is the ability of the system to prevent or resist unauthorized access while providing access to legitimate users. An attack is an attempt to breach security.

(5) Testability refers to the ease with which the software can be made to demonstrate its faults or lack thereof. To be testable the system must control inputs and be able to observe outputs.

(6) Usability is how easy it is for the user to accomplish tasks and what support the system provides for the user to accomplish this.

\subsection{Metrics}

When we are assessing implemented software products, we should consider both product business value and product technical quality.

To assess the business value of a system, stakeholders are interviewed to discuss the use of the system, the business processes that are supported, the system dependability, and the importance of the system outputs.

To assess a software system from a technical perspective, we need to consider both the application system itself and the environment in which the system operates.

The environment is important because many system changes result from changes to the environment, such as upgrades to hardware or operating system. If possible, in the process of environmental assessment, you should make measurements of the system and its maintenance processes. Examples of data that may be useful include the costs of maintaining the system hardware and support software, the number of hardware faults that occur over some time period and the frequency of patches and fixes to the system support software.

To assess the technical quality of an application system, you have to assess a range of factors that are primarily related to the system dependability and the system documentation. You may also collect quantitative system data that will help you judge the quality of the system. Examples of data that might be collected are: the 
number of system change requests, the number of user interfaces, and the volume of data used by the system.

The business value of a legacy system and the quality of the application software and its environment should be assessed to determine whether the system should be replaced, transformed or maintained.

\section{Case Study Background}

The aviation industry has grown at an unprecedented rate.

To cope up with the growth, airports have to expand the terminal facilities and meet new standards of operational efficiency. Airports need software systems that support their ability to evolve in response to their rapidly changing environment. Legacy systems that limit a business's adaptability are seen as significant problems. In response to this situation, airports have started implementing new technologies at the terminals for convenience of the passenger. The new solutions strive to improve operational efficiency and reduce queues at the airport. The new technologies like self-service and web check-in are being installed at many airports to increase Check-in capacity. There are two major check-in systems commonly used by international airports: The Common User Terminal Equipment (CUTE) system; and the Common Use Self Service (CUSS) system [14].

As illustrated in figure 4, with the CUTE system the passenger arrives at the airport and approaches the check-in counter. The check-in process is a one-step process where he/she can interact with the check-in agent and decide on seats and drop bags.With the CUSS system passengers with baggage can drop the bags at the baggage drop-off and proceed to the security check.

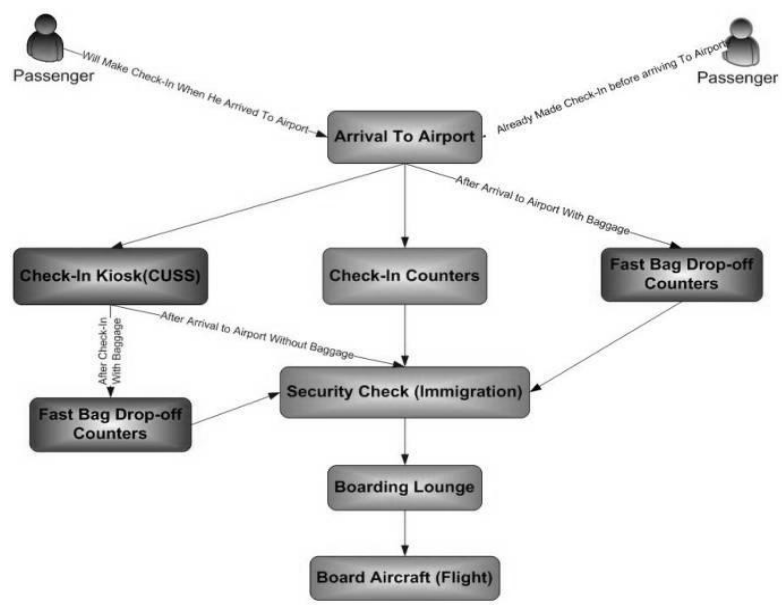

Figure 4: Check-in Context Diagram 
Developing a software Architecture Comparison Analysis Method for Critical Systems at International Airports

\subsection{The CUTE System}

CUTE was 1st implemented in 1984 for the Los Angeles Summer Olympic Games. From 1984 until the present, approximately 400 airports worldwide have installed some level of CUTE. CUTE systems allow an airport to make gates and ticket counters in common use. These systems are known as "agent- facing" systems, because they are used by the airline agents to manage the passenger check-in and boarding process. Whenever an airline agent logs onto the CUTE system, the terminal is reconfigured and connected to the airline's host system. From an agent's point of view, the agent is working within his airline's information technology (IT) network.

The Air Transport Association (IATA) describes the factors to be considered for the design of the check-in area for the check-in desks with CUTE. IATA provides some standard thumb rules based on the queuing theories which are very useful in sizing the overall terminal at the initial stages.

\subsection{The CUSS System}

This evolving pattern enables passengers to obtain boarding passes, check baggage, and conduct other transactions at times and places of their convenience. Passenger check in procedures will gradually shift from check in procedures performed at check in counters, to check in procedures performed at home through the internet, by mobile phone, or through self service check in facilities at the airport such as CUSS Kiosk.

The trend is towards common use equipment which may consist of free standing column type or counter type workstations with built-in Automated Ticket and Boarding pass (ATB) printer. The CUSS provides ticketed passengers the ability to perform many tasks including, but not limited to, check-in for flights, selecting or changing a seat assignment, and obtaining a boarding pass for their departures. The CUSS will be used by self-service passengers to check-in, allocate a seat, print a boarding pass, and baggage check-in in a common use environment. Self-service is becoming the common check-in mechanism in Europe, US and in many airports. In the MEA-Middle East Area region it started as a dedicated self-service and the first CUSS kiosks have been installed at Cairo Airport International TB3. The CUSS is designed for the use of different types of passengers with or without luggage where passengers with luggage could use the new facility of the Common Use Baggage System. The CUSS platform software is responsible for managing the entire Kiosks System, The final configuration of the CUSS kiosk varies depending on the airport operational and security requirements. The equipment required for CUSS consists of two redundant servers (usually the same servers used for CUTE system), located in the MER - Main Equipment Room and self service kiosks. 


\section{Experience at Cairo International Airport}

We have developed an experiment at Cairo International Airport (CAI) to compare between the two check-in systems (the CUTE system and the CUSS system) discussed in section 4 . To assess the business value and the technical quality, a survey has been elaborated and operational metrics have been collected for both systems that are deployed by Egypt Air Airlines.

The assessed quality attributes and the metrics used are listed in table 1:

Table 1: Experiment quality attributes and used metrics

\begin{tabular}{|l|l|}
\hline Quality Attribute & Used Metrics \\
\hline Passenger Satisfaction & $\begin{array}{l}\text { - Process Time } \\
\text { - Queuing Time }\end{array}$ \\
\hline System reliability & Failure rates during 6 months \\
\hline Service availability & Uptime during one month \\
\hline
\end{tabular}

\section{5-1 Measuring Customer Satisfaction}

From the survey the majority of passengers consider the CUSS System process faster than the CUTE System as shown in Table 2 with $68 \%$ strongly agree versus $10.75 \%$ for the CUTE System.

Table 2: Rate of Process Speed

\begin{tabular}{|c|c|c|c|c|c|}
\hline & \multicolumn{5}{|c|}{ Process Speed } \\
\hline & 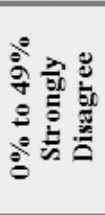 & 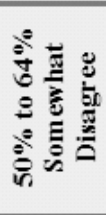 & 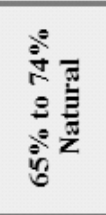 & 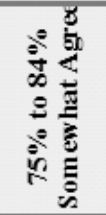 & 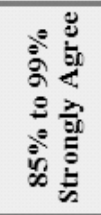 \\
\hline CUTE & $23.42 \%$ & $18.99 \%$ & $25.32 \%$ & $21.52 \%$ & $10.75 \%$ \\
\hline CUSS & $0 \%$ & $0 \%$ & $8 \%$ & $24 \%$ & $68 \%$ \\
\hline
\end{tabular}


Also the survey indicates that the majority of passengers are satisfied with CUSS "Change/Select seat" service as shown in Table 3 with 92\% strongly agree versus $0 \%$ for CUTE.

Table 3: Rate of passenger satisfaction

\begin{tabular}{|c|c|c|c|c|c|}
\hline & \multicolumn{5}{|c|}{ Change/ Select Seat } \\
\hline & 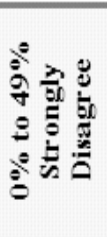 & 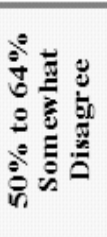 & 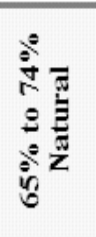 & 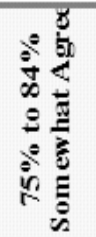 & 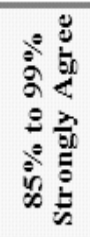 \\
\hline CUTE & $0.00 \%$ & $0.00 \%$ & $0.00 \%$ & $0.00 \%$ & $0.00 \%$ \\
\hline CUSS & $0 \%$ & $0 \%$ & $4 \%$ & $4 \%$ & $92 \%$ \\
\hline
\end{tabular}

Check-in counters were used by Egypt Air Airlines and there were a maximum of six counters open at the time of observation. As shown in figure 5, the average processing time per passenger for CUTE System is 2.74 minutes. CUTE's average processing time is smaller than the CUSS System. This is a result of the efficiency of the check-in agent and the interaction with the passenger. This human element causes significant variations in check-in times that are shown in figure 5. However, the comparison between CUSS without bags and CUTE indicates clearly that CUSS' processing time is faster than the CUTE as illustrated in figure 6. The standard deviation in processing time is shown in figure 7.

The standard deviation for CUTE System is 1.7 while that of CUSS without bag is 1.3 and the Total (CUSS+BAG) is 2.3.

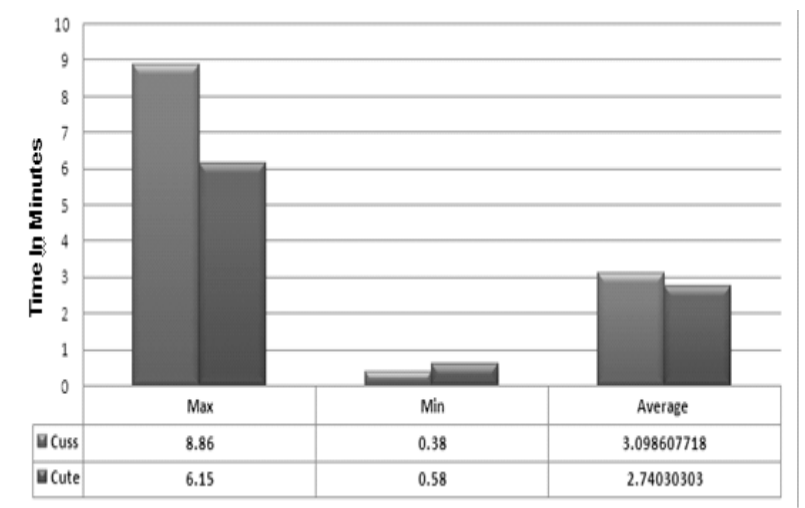

Figure 5: Comparison between CUSS with Bags and CUTE 


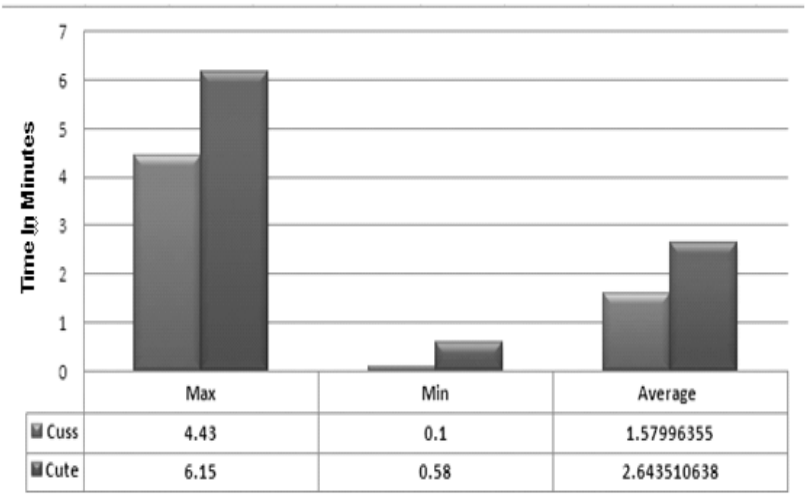

Figure 6: Comparison between CUSS without Bags and CUTE

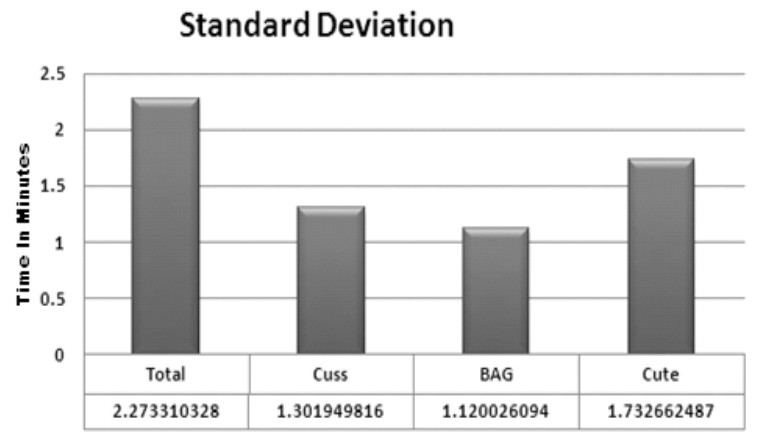

Figure 7: Standard Deviation of Process Time

As shown in Table 4 and figure 8, the processing time for CUTE is often between one and two minutes per passenger while CUSS takes less than a minute.

Passengers using kiosks need to go to baggage drop-off if required to check in bags. The processing time for each process is shown in Table 4. The characteristics for each method are discussed in detail in this section.

Table 4: All ranges of processing time for all Check-In System

\begin{tabular}{|c|c|c|c|c|c|c|c|c|c|}
\hline & $0-1$ & $1-2$. & $2-3$. & $3-4$. & $4-.5$ & $5-6$. & $6-.7$ & $7-8$. & $8-9$. \\
\hline CUTE & 1 & 49 & 9 & 10 & 13 & 11 & 6 & 0 & 0 \\
\hline CUSS & 40 & 27 & 13 & 10 & 9 & 0 & 0 & 0 & 0 \\
\hline Bag & 30 & 35 & 17 & 12 & 5 & 0 & 0 & 0 & 0 \\
\hline Total & 12 & 29 & 22 & 7 & 10 & 3 & 10 & 1 & 5 \\
\hline
\end{tabular}




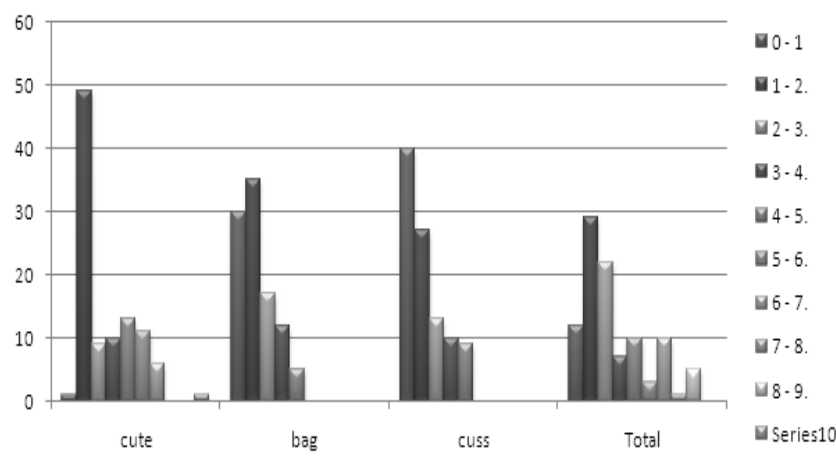

Figure 8: All ranges of processing time for all Check-In Systems

\subsection{Analyzing Failure Rates}

Failure rate is the frequency with which an engineered system or component fails, expressed for example in failures per hour. It is often denoted by the Greek letter $\lambda$ (lambda) and is important in reliability engineering.

The failure rate of a system usually depends on time, with the rate varying over system age. The measurement of failure rates (hardware, software) for all workstation using CUTE or CUSS Systems for 6 months is shown in figure 8 .

\section{Failure Rate}

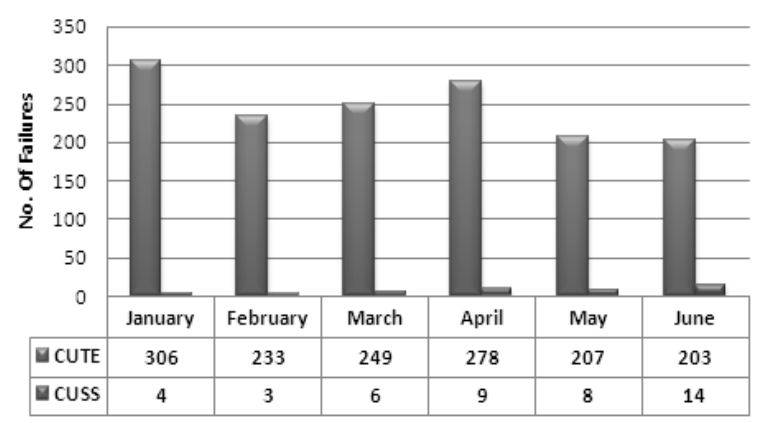

Figure 8: The Failure rate of Check-In System

\subsection{Analyzing System Service Availability}

Availability means that a system is on-line and ready for access. A variety of factors can take a system off-line, ranging from planned downtime for maintenance to catastrophic failure. The goals of high availability solutions are to minimize this downtime and/or to minimize the time needed to recover from an 
outage. Exactly how much downtime can be tolerated will dictate the comprehensiveness, complexity and cost of the solution.

The following steps are used to measure availability.

Step1: Collecting and Presenting system service availability data for each checkin system (CUTE and CUSS) for one month as shown in Figure 9.

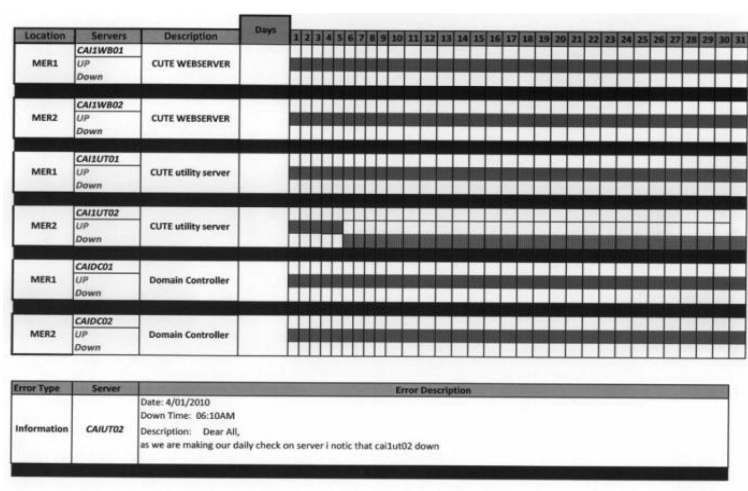

Figure 9: Time Chart for CUTE/CUSS System in January 2010

Step2: Calculate the failure rate data for each server in each system in each month as shown in table 5 .

These systems require processing, 24 hours a day, 7 days a week; it means these systems require $24 \times 7$ availability with $99.9 \%$ uptime.

Table 5: All Servers of Check-In Systems

\begin{tabular}{|cccc|}
\hline System & Server Name & Houlr & failure \\
\hline CUSS & CA11WB01 & 744 & No Failure \\
\hline CUSS & CA11WB02 & 744 & No Failure \\
\hline CUTE & CA11UT01 & 744 & No Failure \\
\hline CUTE & CA11UT02 & 96.72 & Failure \\
\hline
\end{tabular}

$\frac{1 \text { Failure }}{840.72 \text { Hours }}=0.001198$

or 0.001198 failures for every 744 hours of operation 
Developing a software Architecture Comparison Analysis Method for Critical Systems at International Airports

\subsection{Queuing Time}

Queuing theory deals with the mathematics involved in the study of waiting lines, or queues. The basis of the problem is a customer that arrives and joins a queue waiting for service, and then he receives the service and exits. To study queuing systems, information is required on the arrival process, the service process and the queue discipline. The arrival process deals with how arrivals are distributed over time. Most of the work in queuing theory has revolved around a Poisson distribution; this means that the inter-arrival time between successive arrivals is unrelated and exponentially distributed.

The service process requires information on the service time; the number of servers and whether the servers are in series or parallel again a Poisson distribution is commonly used.

The queue discipline deals with how items are chosen from the queue for service. Common rules are FIFO (First In First Out) and LIFO (Last In First Out). Other considerations are the capacity of the queue, customers avoiding the queue due to length and customers leaving the queue due to waiting too long.

Queuing theory answers questions about the system such as: a customer's mean waiting time in the queue, a customer's mean time in the system, and the length of the queue or server utilization. With this knowledge, changes to the system can be investigated; such as implementing additional servers, prioritizing customers or adjusting the size of the waiting area.

\subsubsection{M/M/1 Model}

$\mathrm{M} / \mathrm{M} / 1$ is Kendall's notation of this queuing model. The first part represents the input process, the second represents the service distribution, and the third represents the number of servers as illustrated in figure 10.

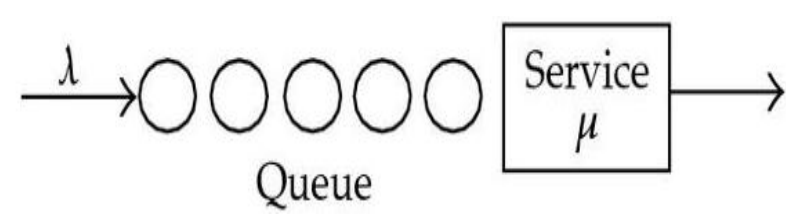

Figure 10: one server one line queue system (M/M/1)

The $M$ represents an exponentially distributed inter-arrival or service time; specifically $M$ is an abbreviation for Markovian. The M/M/1 Waiting line system has a single channel, single phase, Poisson arrival rate, exponential service time, unlimited population, and First-in First-out queue discipline. For an $\mathrm{M} / \mathrm{M} / 1$ queue where $\lambda$ is average arrival rate into the system and $\mu$ is the average 
service rate, it is simple to calculate questions about the system. The average number of customers in the system is given by (1), and the total time in the system is given by (2). Similar equations have been developed for variations on the $\mathrm{M} / \mathrm{M} / 1$.

$$
\begin{aligned}
& \frac{\rho \text {-1 }_{\text {Where }}}{\rho-\mu} \rho=\frac{\lambda}{\mu} \\
& \frac{1}{\lambda-\mu}
\end{aligned}
$$

The input parameters to the queuing system is illustrated in table 6 .

Table 6: Input parameter to queuing Time system

\begin{tabular}{|l|l|}
\hline \multicolumn{1}{|c|}{ Input Parameter } & \multicolumn{1}{l|}{ The value } \\
\hline Arrival Rate $(\boldsymbol{\lambda})$ & 6 \\
\hline Service Rate $(\boldsymbol{\mu})$ & 8 \\
\hline Experiment duration & 3600 \\
\hline Maximum queue length & 100 \\
\hline
\end{tabular}

This simulated experiment examines the MM1 queuing system and here is the explanation for the above table input data:

1) Arrival Rate $(\lambda)=6$ Passengers per Minute.

2) Service Rate $(\mu)=8$ Serviced Passengers per Minute.

3) Experiment Duration $=3600$ Minute $=60$ Hour

4) Maximum Queue Length= 100 Passenger in System Waiting “Area” Queue The results are illustrated in table 7 .

\begin{tabular}{|c|c|c|}
\hline Result & Computed value & Simulated value \\
\hline Customers in system $\left(L_{s}\right)$ & 3 & $3.0102030:$ \\
\hline Customers in queue $\left(\mathrm{L}_{\mathrm{q}}\right)$ & 2.25 & 2.2567900 \\
\hline Time in system $\left(W_{s}\right)$ & 0.5 & $0.5022366:$ \\
\hline Time in queue $\left(W_{a}\right)$ & 0.375 & $0.3765161:$ \\
\hline Idle probability $\left(\mathrm{p}_{0}\right)$ & 0.25 & 0.24969871 \\
\hline Server utilization $(\rho)$ & 0.75 & $0.7534130:$ \\
\hline
\end{tabular}

Table 7: Basic Result

The computed results came from the formulas of MM1 queuing system, and the simulated results come by experimental approach from random sample of space.

And the results are satisfying mostly if we knew that the:

1) Passengers in system (Ls) $=3.01$ at the same time taking service 
Developing a software Architecture Comparison Analysis Method for Critical Systems at International Airports

2) Passengers in queue $(\mathrm{Lq})=2.256$ passenger waiting to start being serviced.

3) Time in system (Ws) $=0.502$ to finish the service.

4) Time in queue $(\mathrm{Wq})=0.376$ waiting time inside the passengers waiting area.

5) Idle probability $(\mathrm{p} 0)=0.249$ the probability that the system server is being idle.

6) Server utilization $(\rho)=0.753$ which lead to a great performance on the other hand.

\subsection{Summary}

From the results mentioned above, we can deduce the following: The passenger is satisfied with the process of self-service check-in using CUSS Kiosk, the process of self-service is faster, more accurate and easier to understand. Self-service puts control into the hands of the customer.In the airline industry, this control comes in the form of enabling the customer to select his own seat, request an upgrade, or change flights.

Further to this conclusion, the following was observed:

1) The minimum time was 0.38 minutes and on average was 3.1 minutes to complete a transaction and print a boarding pass from CUSS System.

2) The processing times for the passenger who had some experience of using a kiosk was significantly less than average.

3) Most of the passengers need assistance in completing the process and there were two roving agents helping passengers.

4) The location of the kiosks made them very accessible and easily visible before the passengers could see the check-in counters.

\section{Conclusion and Future Work}

This paper discusses the need to adapt the Software Architecture Comparison Analysis Method -SACAM in order to use it in legacy software modernization context, where candidate implemented software products are evaluated.

The main contribution of this paper is proposing an adaptation by considering the evolution/maintenance history in architecture evaluation, and introducing complementary techniques that allow assessing software products from both business value perspective and technical quality perspective. The proposed adaptation was demonstrated by conducting a comparison between two check-in systems used in international airports as a case study.

The future work includes extending the method to apply in other system modernization situations such as reengineering and the comparison between an existing product and a planned one. 


\section{References}

[1] Ian Sommerville, (2007), Software Engineering Eighth Edition, AddisonWesley(Ch. 3).

[2] Laprie, J.C., (1995), Dependable Computing: Concepts, Limits, Challenges, Proc. 25th IEEE Symposium on Fault-Tolerant Computing.

[3] Thayer, R.H., (2002), Software System Engineering: a tutorial IEEE Computer (Ch.2).

[4] Banani Roy and T.C. Nicholas Graham, (2008), Methods for Evaluating Software Architecture: A Survey, Technical Report No. 2008-545, School of Computing, Queen's University at Kingston, Ontario, Canada

[5] Christoph Stoermer, Felix Bachmann, Chris Verhoef, (2003), SACAM: The Software Architecture Comparison Analysis Method, TECHNICAL REPORT, CMU/SEI-2003-TR-006, ESC-TR-2003-006.

[6] Clements, P.; Kazman, R.; \& Klein, M., (2002), Evaluating Software Architectures. Reading, MA: Addison Wesley.

[7] Barbacci, M.; Ellison, R.; Lattanze, A.; Stafford, J.; Weinstock, C.; \& Wood, W., (2003), Quality Attribute Workshops (QAWs), Third Edition (CMU/SEI2003-TR-016).

[8] Bachmann, F.; Bass, L.; \& Klein, M., (2003), Deriving Architectural Tactics: A Step Toward Methodical Architectural Design (CMU/SEI-2003-TR-004, ADA413644

[9] Bass, L.; Clements, P.; \& Kazman, R., (2003), Software Architecture in Practice, Second Edition. Reading MA: Addison Wesley.

[10] Arora, V.; Kalaichelvan, K.; Goel, N.; \& Munikoti, R., (1995), Measuring High-Level Design Complexity of Real-Time Object-Oriented Systems, Proceedings of the Annual Oregon Workshop on Software Metrics.

[11] Faust, D. \& Verhoef, C., (2003), Software Product Line Migration and Deployment, Software: Practice and Experience, Volume 33, Issue 10.

[12] Clements, P.; Bachmann, F.; Bass, L.; Garlan, D.; Ivers, J.; Little, R.; Nord, R.; \& Stafford, J., (2002), Documenting Software Architectures Views and Beyond. Reading, MA: Addison Wesley.

[13] Stoermer, C.; O’Brien, L.; \& Verhoef, C., (2003), Moving Towards QualityAttribute-Driven Software Architecture Reconstruction, Proceedings of the 10th Working Conference on Reverse Engineering (WCRE) Victoria, Canada. 
Developing a software Architecture Comparison Analysis Method for Critical Systems at International Airports

[14] Shady G. Abdelaziz, Abdelfatah A .Hegazy and Ahmed Elabbassy , (2010), Study of Airport Self-service Technology within Experimental Research of Check-in Techniques Case Study and Concept, IJCSI International Journal of Computer Science Issues, Vol. 7, Issue 3, No 1.

[15] G. Abowd, L. Bass, P. Clements, Rick Kazman, L. Northrop, and A. Zaremski, (1996), Recommended Best Industrial Practice for Software Architecture Evaluation (CMU/SEI-96-TR-025). Pittsburgh, Pa.: Software Engineering Institute, Carnegie Mellon University.

[16] M. A. Babar, L. Zhu and R. Jeffery, (2004), A Framework for Classifying and Comparing Software Architecture Evaluation Methods, In the Proceedings on Australian Software engineering, pp. 309-318.

[17] M. Lindvall, R. T. Tvedt and P. Costa, (2003), An empirically-based process for software architecture evaluation, Empirical Software Engineering VOL.8, No.1.

[18] R.T. Tvedt, M. Lindvall, and P. Costa, (2002), A Process for Software Architecture Evaluation using Metrics, In the proceedings of 27th Annual NASA Goddard/IEEE.

[19] Davide Falessi1, Muhammad Ali Babar2, Giovanni Cantone1, Philippe Kruchten3, (2009), Applying Empirical Software Engineering to Software Architecture: Challenges and Lessons Learned, Technical Report 09.80, Dipartimento Di Informatica, Sistemi E Produzione, Universita Degli Studi Di Roma "Tor Vergata".

[20] Eric Bouwers, Joost Visser, Arie van Deursen, (2009), Criteria for the Evaluation of Implemented Architectures, ReportTUD-SERG-2009-018, Delft University of Technology, Software Engineering Research Group. Accepted for publication in the Proceedings of the International Conference on Software Maintenance (ICSM), 2009, IEEE Computer Society

[21] Muhammad Ali Babar, Patricia Lago, Paris Avgeriou, (2009), Empirical Assessment in Software Architecture: Importance and Challenges, Wicsa7 Workshop:Empirical

Assessment in Software Architecture.

[22] Marwan Abi-Antoun, Talia Selitsky, Thomas LaToza, (2010), Developer Refinement of Runtime Architectural Structure, SHARK'10 May 2, 2010, Cape Town, South Africa. 
[23] Michael Mattsson, Håkan Grahn, and Frans Mårtensson, (2006), Software Architecture Evaluation Methods for Performance, Maintainability, Testability, and Portability, Second International Conference on the Quality of Software Architectures (QoSA 2006), Västerås, Sweden.

[24] Antonia Bertolino1, Henry Muccini2, and Andrea Polini1, (2006), Architectural Verification of Black-box Component-Based Systems, RISE'06 Proceedings of the 3rd international conference on Rapid integration of software engineering techniques.

[25] Komaldeep Purewal, Dr. Lili Yang, Dr. Alan Grigg, (2009), Quantitative Assessment of Quality Attributes in Systems Architecture using Evidential Reasoning, 7th Annual Conference on Systems Engineering Research (CSER 2009).

[26] L. Bass, P. Clements, and R. Kazman, (1998), Software Architecture in Practice. Reading, Mass.: Addison-Wesley. 